\title{
The intrinsic structure of hydrophobic surfaces: the oil-water interface
}

\author{
Fernando Bresme ${ }^{1}$, Enrique Chacón ${ }^{2}$, Pedro Tarazona ${ }^{3}$ and Kafui Tay ${ }^{1,4}$ \\ 1 Department of Chemistry, Imperial College London SW7 2AZ, London UK \\ 2 Instituto de Ciencia de Materiales de Madrid, CSIC, Madrid Spain \\ 3 Departamento de Física Teórica de la Materia Condensada, \\ Universidad Autónoma de Madrid, Madrid Spain and \\ ${ }^{4}$ Laboratoire de Chimie Physique, Université de Paris-Sud 11, 91405 Orsay Cedex, France.*
}

(Dated: June 24, 2008)

\begin{abstract}
We investigate the water-oil interface using molecular dynamics simulations of realistic models of alkanes and water. The intrinsic density profiles are computed using a methodology that removes the smoothing effect of the capillary waves. We show that at $300 \mathrm{~K}$ the intrinsic width of the gap separating the oil and water phases spans little more than one water molecule diameter, and undergoes very weak short-ranged fluctuations, indicating that the water-oil interface is a rigid molecular structure at ambient temperature. Only near the drying transition (above $500 \mathrm{~K}$ for dodecane), the gap features uncoupled fluctuations of the oil and water surfaces, as expected in a typical drying structure. We find that the intrinsic structure of water next to the oil phase is remarkably similar to the bare water-vapor interface.
\end{abstract}

PACS numbers: 68.03.Hj, 68.03.Kn, 68.05.Cf, 82.70.Uv

Understanding the properties of water next to hydrophobic surfaces is essential to develop a microscopic description of biological macromolecules and materials in solution. The oil-water interface is a particularly useful model in this instance, since the structural changes undergone by water at this hydrophobic surface are expected to be similar to those found at biological and materials surfaces. Stillinger suggested that the interfacial structure of water at a "flat repelling" hydrophobic surfaces should be similar to that of the water-air interface [1]. An experimental proof of this idea is far from trivial. Modern spectroscopic and diffraction techniques provide a powerful approach to investigate buried interfaces, such as those appearing in hydrophobic materials. As a matter of fact, sum frequency spectroscopy (SFS) experiments of the alkane-water interface have suggested that water hydrogen bond interactions at the alkane surface are weaker than at the corresponding liquid-vapor interface [2]. Moreover X-ray and neutron diffraction techniques have been used to investigate the oil-water interface [3, 4]. One of these works [3] has suggested that the intrinsic structure of the alkane-water interface could add a significant contribution to the average density profile.

The global structure of water at a hydrophobic surface has been discussed in terms of the so called depletion layer. Water depletion is one element of recent theories of hydrophobic forces $[5,6]$. Moreover, capillary drying between hydrophobic materials has been put forward as an explanation of the hydrophobic force in extended hydrophobic surfaces [7]. This idea has raised a hot controversy (see for instance $[5,8-11]$ ).

Recent experiments [12-14] provide support for the for-

*Electronic address: f.bresme@imperial.ac.uk, e.chacon@icmm. csic.es, pedro.tarazona@uam.es, kafui.tay@lcp.u-psud.fr mation of a narrow depletion layer at hydrophobic surfaces. The width has been reported to be of the order of 1-6 $\AA$ [12], whereas the density of the depletion layer appears to be about $40-70 \%$ [12] of the bulk water density. The two most recent X-ray reflectivity experiments [12] show, within the experimental resolution, that the depletion layer is insensitive to dissolved gases, and both works discard nanobubble nucleation as an explanation of the experimental results. These two observations are in contradiction with earlier experimental work [15].

The discussion above shows that the investigation of water next to hydrophobic surfaces is an extremely active, as well as controversial, area of research. The existence or absence of a drying surface phase transition, as well as different aspects of the structure of water at liquid-liquid interfaces have been addressed both in theoretical and computer simulation studies $[6,7,9,16,17]$. In particular the quantification of the fluctuations undergone by the interface represents a major challenge. These fluctuations are expected to be significant in capillary drying processes, solvent reorganization and kinetics of self assembly. Computer simulations of the oil/water interfaces may be used to answer these questions, but the interfacial profiled are affected by capillary wave (CW) fluctuations, which depend on the interface sampling area [18]. Hence, an approach that eliminates the blurring effect of the $\mathrm{CW}$ is highly desirable as a means to access essential molecular information of the interfacial structure. In this paper we provide direct information on the intrinsic width, intrinsic structure, and fluctuations of the oil-water interface.

We have performed molecular dynamics simulations with realistic models of the oil-water interface. The interface was constructed by putting together two preequilibrated liquid slabs of n-alkane and water [19], surrounded by their corresponding vapor phases to ensure coexistence conditions for both liquids. In this paper, 
oil (dodecane or hexane) is modeled using a united atom approach and the TraPPE forcefield [20], and water is simulated using the SPC/E model [21]. The long range coulombic interactions were handled using the Ewald summation method. The short range water-alkane interactions were derived using standard combining rules, and the potentials were truncated at $6.2 \sigma$, where $\sigma=$ $3.166 \AA$, is the diameter of the oxygen atom in the SPC/E model. All the simulations were performed in prismatic boxes with dimensions, $L_{x}=L_{y}=13 \sigma$ and $L_{z}=100 \sigma$. The cross sectional area of the interface $A=L_{x}^{2}$ was chosen to minimize periodic boundary effects [22]. The liquid slab thicknesses, $\approx 50 \sigma$ for oil and $\approx 20 \sigma$ for water, were chosen to avoid correlations between different interfaces. A typical simulation involved about $N=17400$ particles, 600 alkanes and 3400 water molecules. The equilibration time was of the order of $1 \mathrm{~ns}$ and the production time of 2-3 ns, with a timestep of 2 femtoseconds. The density profiles, and other properties of the oil-water $(\mathrm{o} / \mathrm{w})$ interface are labeled as $\rho_{\alpha}(z)$, using the subscript $\alpha=\mathrm{o}, \mathrm{w}$ to indicate the different species; while those of the (oil or water) free liquid-vapor surface are denoted by $\rho_{\alpha}^{\mathrm{v}}(z)$.

We have used the intrinsic sampling method (ISM) developed in ref. [23] to compute both the intrinsic density profile, $\tilde{\rho}_{\alpha}(z)$, and the relevant statistics for the position and fluctuations of the intrinsic surface (IS), $z=\xi_{\alpha}(\mathbf{R})$, defined as the minimal area surface going through the outmost molecular layer defined by atoms of species $\alpha$. The CW fluctuations within our simulation box are measured by the mean square $\mathrm{CW}$ amplitude $\Delta_{\alpha} \equiv\left\langle\xi_{\alpha}^{2}\right\rangle-\left\langle\xi_{\alpha}\right\rangle^{2}$; e.g. for the dodecane-water interface at $300 \mathrm{~K}$ we find $\Delta_{\mathrm{w}}=0.18 \sigma^{2}$ and $\Delta_{\mathrm{o}}=0.15 \sigma^{2}$. These values would grow with the transverse size of the box $[18,26]$, but already within our area $A$ the blurring effect of these CW fluctuations result in smooth density profiles (c.f. Fig. 1) that hide the intrinsic layering structure of $\tilde{\rho}_{\alpha}(z)$ (see also ref. [24] for a similar although more limited approach). Figure 1 shows the intrinsic profile of the oil-water interface. This can be compared with the intrinsic profile of the SPC/E liquid-vapor interface that has been reported recently [25]. Our simulations, both for dodecane and hexane [26], show that the intrinsic profile is essentially independent of the presence of a hydrophobic surface. This result validates, at a quantitative level, the notion that the water structure next to a hydrophobic surface is very similar to that of the water-air interface [1]. We would like to note that this agreement between both liquid-water and water-vapor interfaces is not connected to the proximity of the thermodynamic state to the drying transition (see below).

The dipolar and tensorial distributions for the intrinsic orientations of the water molecules (c.f. Fig. 2) provide a more sensitive test to analyze the influence of the environment on the structure of interfacial water. Negative values of $T(z)=\left\langle 3 \hat{u}_{\mathrm{z}}^{2}-1\right\rangle / 2$ indicate a tendency of the dipole vector $\hat{\mathbf{u}}$ to be preferentially flat on the surface. We find that the preference for this orientation is $\approx 20 \%$ higher at the $\mathrm{o} / \mathrm{w}$ interface than at the free liquid surface.

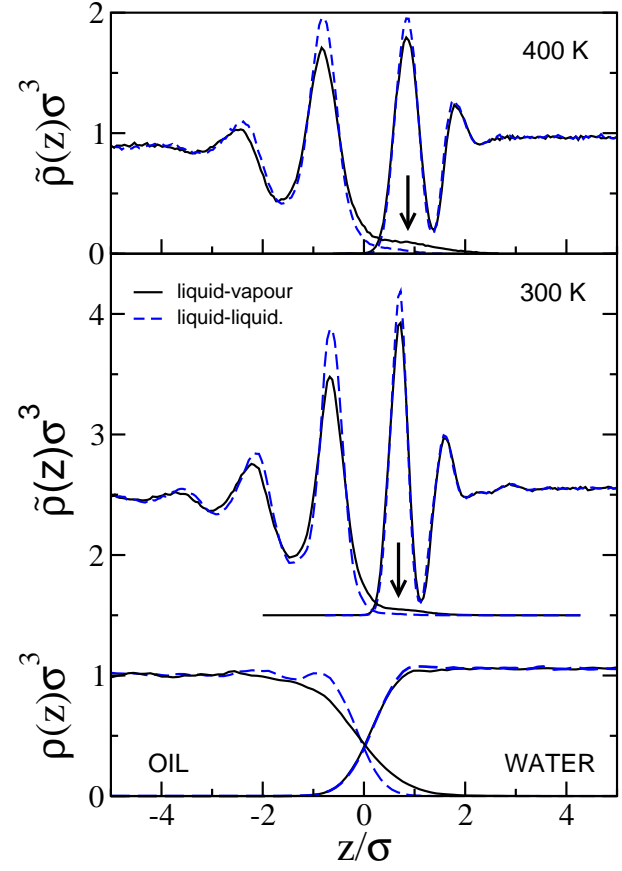

FIG. 1: Intrinsic density profiles of water (oxygen atoms) and dodecane (all carbon pseudoatoms) for liquid-vapor (full lines) and liquid-liquid interfaces (dashed lines) at 300 and $400 K$. The mean density profiles are presented for the lower $T$. The arrows signal the tail of the hydrocarbon layers in direct contact with the water surface.

The fluctuations of the dipole $\hat{\mathbf{u}}$ out of the surface plane are asymmetric, with a tendency to point away from the liquid bulk $\left(p(z)=\left\langle\hat{u}_{z}\right\rangle \leq 0\right)$ at the external side of the outmost layer (i.e. at the extrusions of the water surface), and the inverse tendency at the inner part of that layer (i.e. at the surface intrusions) [25]. These orientational features are compatible with the existence of dangling bonds at the interface [19]. It is remarkable that the presence of the oil phase does not change the mean orientation of the most external water molecules, but modifies ( $\approx 20 \%$ increase) the molecular orientations of water at the inner part of the outmost layer.

In order to investigate the intrinsic profile of the alkanes we have extended the ISM to treat flexible molecules. A full description of the methodology will be reported elsewhere. Briefly, our method identifies the outmost molecular layer in terms of blocks of three consecutive carbon pseudoatoms along the chain. This enables us to distinguish the alkane molecules lying flat on the interface, and hence contributing to the IS with all their carbon pseudoatoms, from those where only part of the molecule contributes to the outmost molecular layer. We keep the same level of resolution for $\xi_{\mathrm{o}}(\mathbf{R})$ and $\xi_{\mathrm{w}}(\mathbf{R})$, with a $\mathrm{CW}$ length larger than $1.8 \sigma$, which was found to be optimal for the intrinsic structure of the free water surface [25].Figure 1 reports the intrinsic profiles for the $\mathrm{o} / \mathrm{w}$ and oil-vapor interfaces. The dodecane interface exhibits a stronger dependence with the environment than 


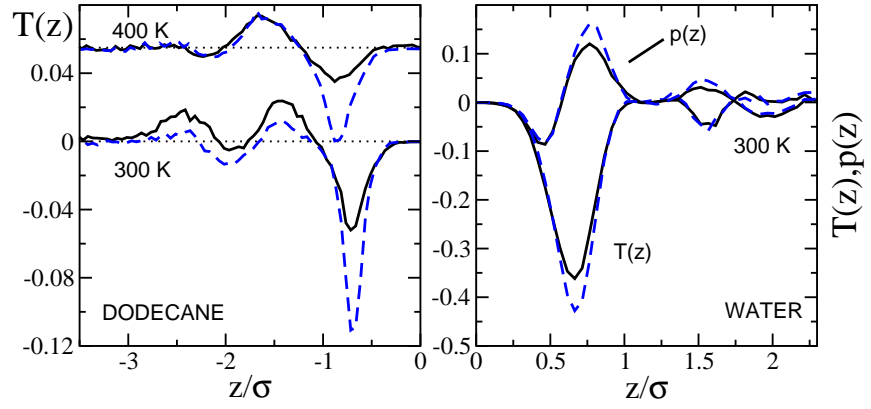

FIG. 2: Intrinsic orientational order parameters for (left) dodecane $(T(z)$, at two temperatures) and (right) water $(T(z)$, and the dipole $p(z)$ at the lower temperature). The order parameter for dodecane at $400 \mathrm{~K}$ has been shifted upwards. to facilitate the comparison of the results. Full and dashed lines correspond to the liquid-vapor and liquid-liquid interfaces respectively.

the water interface. Water tends to inhibit the protrusions of the alkane molecules out from its corresponding liquid phase, an effect that is amplified at higher temperatures (see $\tilde{\rho}_{\mathrm{o}}^{\mathrm{v}}(z)$ and $\tilde{\rho}_{\mathrm{o}}(z)$ and arrows in Fig. 1). The alkane groups buried in the liquid (at distances of $\approx 2 \sigma$ ), can sense the water surface. Also we observe surface segregation of the terminal $\mathrm{CH}_{3}$ groups at both the free surface and dodecane-water interface, in qualitative agreement with the SFS experiments of the free dodecane surface [27].

The alkane intrinsic orientational profiles, $T(z)$ (c.f. Fig. 2) represent the orientation of the vector joining the two terminal $\mathrm{CH}_{3}$ groups. Dodecane exhibits a much weaker tendency than water to orient itself at the interface (see magnitude of the order parameter); the dodecane molecules sitting at the first layer tend to lie flat $(T(z)<0)$, whereas those in between the first and second layer tend to lie with their main axis perpendicular to the interface. This alternating behavior is transmitted inside the liquid, but dies away in about $2 \sigma$ diameters. Again, the molecules can sense whether they are at the liquidvapor or the $\mathrm{o} / \mathrm{w}$ interface. In the presence of water, the dodecane molecules occupying the first layer show a larger tendency to adopt parallel orientation with respect to the interface plane. This agrees with the intrinsic density profile (cf. Fig. 1), which indicates a tendency for the water surface to inhibit dodecane protrusions away from the liquid surface. Increasing the temperature to $400 K$ significantly decreases the dodecane orientational order (see Fig.2) at the liquid-vapor surface, but there is still a clear preferred orientation in the outmost layer in the presence of water.

In the following, we investigate the correlations between the dodecane and water surfaces at the $\mathrm{o} / \mathrm{w}$ interface. To tackle this problem we have computed the intrinsic width of the interfacial gap, defined as the distance $d(\mathbf{R})=\xi_{\mathrm{w}}(\mathbf{R})-\xi_{\mathrm{o}}(\mathbf{R})$, for each point over the surface $\mathbf{R}=(x, y)$. The mean value of $d(\mathbf{R})$ over all the molecular configurations analyzed by the ISM (and all

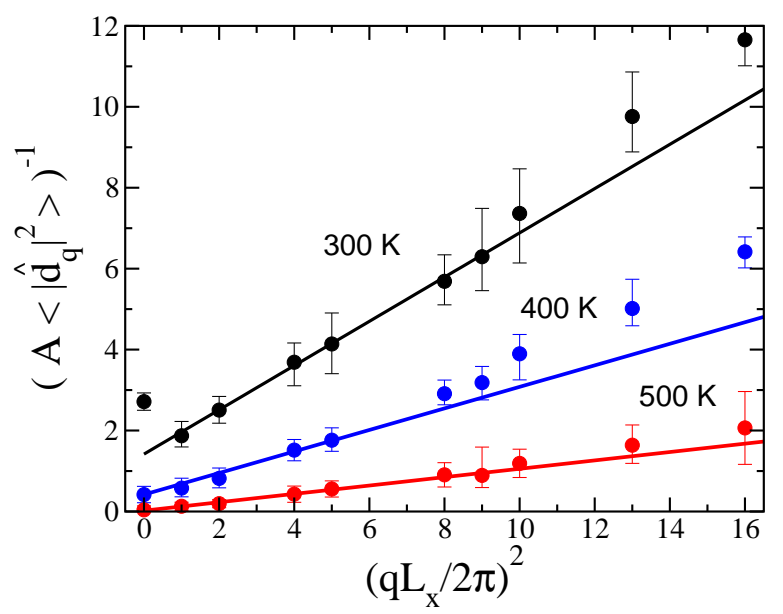

FIG. 3: Inverse dispersion of the fluctuations of the waterdodecane interface as a function of the square of the wave vector. The lines represents a linear fit of the low-q simulation data to equation 1.

the positions $\mathbf{R}$ ) is found to be $\langle d\rangle=1.43 \sigma$ at $300 \mathrm{~K}$, and $1.77 \sigma$ at $400 \mathrm{~K}$, i.e., the gap size is of the order of one water molecule diameter. These values are similar to the thicknesses discussed recently in experiments of water at hydrophobic monolayers [12]. The mean square displacement $\Delta_{d} \equiv\left\langle d^{2}\right\rangle-\langle d\rangle^{2}$ is $0.10 \sigma^{2}$ for the dodecanewater interface at $300 K$, and $0.22 \sigma^{2}$ at $400 K$, which represents about $3 \%$ deviation with respect to the mean value at $300 \mathrm{~K}$, and about $7 \%$ at $400 \mathrm{~K}$. In comparison, the mean square displacements for each individual IS are $\Delta_{\mathrm{w}}=0.18 \sigma^{2}$ and $\Delta_{\mathrm{o}}=0.15 \sigma^{2}$, at $300 K$. Considering uncorrelated fluctuations of $\xi_{\mathrm{o}}$ and $\xi_{\mathrm{w}}$ we would get a mean square displacement $\Delta_{d}=\Delta_{\mathrm{w}}+\Delta_{\mathrm{o}}=0.33 \sigma^{2}$, which is much larger than our value of $0.1 \sigma^{2}$. This result shows that the fluctuation of the two edges of the hydrophobic gap are highly correlated. We note that the logarithmic divergence of $\Delta_{\mathrm{w}}$ and $\Delta_{\mathrm{o}}$ with the interfacial area is eliminated in $d(\mathbf{R})$, since the long-wavelength capillary waves represent rigid local displacements of the whole oil/water interface, without changing the local correlation structure between alkane and water molecules. Therefore $\Delta_{d}$ should have a well defined macroscopic limit for large interfacial area. We expect that only above the drying transition both $\langle d\rangle$ and $\Delta_{d}$ will diverge, as the oil and the water sides of the oil-water interface decouple, with a thick vapor film between them. For dodecane the onset of such surface phase transition is observed in the ISM results at $500 \mathrm{~K}$, with $\langle d\rangle \geq 2.5 \sigma$ and $\Delta_{d} \geq 1.2 \sigma^{2}$. Complete drying is directly observed in the mean density profiles at $550 \mathrm{~K}$.

The intrinsic hydrophobic gap may be further characterized through its Fourier representation, $d(\mathbf{R})=$ $\sum_{q} \hat{d}_{q} \exp (i \mathbf{q R})$, where $\mathbf{q}$, is the wave vector. The mean values of the Fourier components vanish except for $q=0$, $\left\langle\hat{d}_{0}\right\rangle=\langle d\rangle$. The fluctuations of any $\hat{d}_{q}$ decay with increasing transverse area as, $\left\langle\left|\hat{d}_{q}\right|^{2}\right\rangle \sim 1 / A$, but at the 
same time the number of components is proportional to $A$. Hence the sum of all the mean square displacements reproduces the results given above for $\Delta_{d}$. In Fig. 3 we present the inverse values of $A\left\langle\left|\hat{d}_{q}\right|^{2}\right\rangle$, as functions of $\left(q L_{x} /(2 \pi)\right)^{2}$. The low $q$ - fluctuations of the intrinsic hydrophobic gap width can be described by the GinzburgLandau (GL) hamiltonian [18],

$$
\left\langle\left|\hat{d}_{q}\right|^{2}\right\rangle=\frac{k T}{A\left(\kappa+q^{2} \lambda\right)} .
$$

From the fitting to equation (1) we can calculate the transverse correlation length $\zeta=\sqrt{\lambda / \kappa} \approx 1.5 \sigma$, for the local fluctuations of the hydrophobic gap in the dodecane/water interface at $400 \mathrm{~K}$. At lower temperatures $(300 \mathrm{~K})$, the stiffer structure of the interface (lower $\Delta_{d}$ ) results in larger error bars. We also observe a small discrepancy between simulation and theoretical results at $q=0$. The simple form of the GL Hamiltonian may be insufficient to describe the fluctuation spectrum at low temperatures, possibly because the size of the alkane molecules is showing up in the more ordered, low $T$, surface. In any case, even if we consider the largest estimate for the correlation length (shown by the linear fit in Figure 3 ), this would give $\zeta \leq 1.3 \sigma$. The results for $500 \mathrm{~K}$ in Fig. 3 show the proximity to the drying phase transition, where the coefficient $\kappa$ vanishes. Within the finite size and sampling times of our simulations we observe that the transverse correlation length for the gap width increases up to $\zeta \approx 3-5 \sigma$. This value is close to $L_{x} / 2$ in our simulation box, so that it has to be interpreted as the signature of the drying surface phase transition. Similar results are obtained for the hexane/water interface [26]. At $300 \mathrm{~K}$ the characteristics of the oil-water gap are in this case $\langle d\rangle=1.41 \sigma, \Delta_{d}=0.32 \sigma^{2}$ and $\zeta=1.4 \sigma$.

To conclude, our analysis of the intrinsic profiles of the oil/water interface indicates that at room temperature the intrinsic structure of water is very similar to that of the free water surface, despite the fact that the system is far from the drying transition. The intrinsic gap between water and oil surfaces is a rather 'rigid' structure, its width being slightly larger than the water molecular diameter, and with corrugations well below that size. There is no evidence of nanometric bubbles or any other mesoscopic fluctuation until the drying transition is reached around $500 K$ for dodecane. We emphasize the usefulness of the ISM method, providing direct access to the hydrophobic gap width and its fluctuations over the whole wavelength range. We hope that our results will help to clarify some outstanding issues on the structure of water at hydrophobic surfaces, in particular, the role of interfacial fluctuations in the kinetics of self assembly of hydrophobic materials and biomolecules in water.

FB would like to thank the Royal Society for financial support. FB and KT thank EPSRC for funding. EC and PT thank the support of the Dirección General de Investigación, Ministerio de Ciencia y Tecnología of Spain, under grant FIS2004-05035-C03, and the Comunidad Autónoma de Madrid under grant S-0505/Esp0299. The simulations were performed at the Imperial College High Performance Computing Service.
[1] F.H. Stillinger, J. Solution Chem. 2141 (1973).

[2] M.G. Brown et. al., J. Phys. Chem. B, 107237 (2003).

[3] D.M. Mitrinović et. al., Phys. Rev. Lett. 85582 (2000).

[4] A. Zarbakhsh, J. Bowers and J.R.P. Webster, Langmuir 2111596 (2005)

[5] H. Ashbaugh and L. Pratt, Rev. Mod. Phys. 78159 (2006).

[6] L.R. Pratt and A. Pohorille, Chemical Reviews 1022671 (2002).

[7] K. Lum, D. Chandler, and J.D. Weeks, J. Phys. Chem. B 1034570 (1999).

[8] D. Chandler, Nature 437640 (2005)

[9] L.R. Pratt et al., J. Am. Chem. Soc. 1272808 (2005).

[10] F. Bresme and A. Wynveen, J. Chem. Phys. 126044501 (2007).

[11] P. Ball, Chem. Rev. 108, 74 (2008).

[12] A. Poynor et. al., Phys. Rev. Lett. 97266101 (2006);M. Mezger et. al., Proc. Natl. Acad. Sci. USA 10318401 (2006).

[13] M. Maccarini et. al., Langmuir 23598 (2007).

[14] T.R. Jensen et.al., Phys. Rev. Lett. 90086101 (2003).

[15] J.G.W. Tyrrell and P. Attard, Phys. Rev. Lett. 87 176104 (2001); R. Steitz et. al., Langmuir 192409 (2003); D.A. Doshi et. al. Proc. Natl. Acad. Sci. USA 1029458
(2005).

[16] S. Senapati, and M.L. Berkowitz, Phys. Rev. Lett. 87 176101 (2001).

[17] A. Pohorille and M.A. Wilson, J. Molec. Struct (Theochem) 284271 (1993).

[18] see e.g. R. Evans, Adv. Phys. 28143 (1979).

[19] K.A. Tay and F. Bresme, J. Am. Chem. Soc. 12814166 (2006).

[20] M.G. Martin and J.I. Siepmann, J. Phys. Chem. B 102 2569 (1998).

[21] H.J.C. Berendsen, J.R. Grigera, and T.P. Straatsma, J. Phys. Chem. 916269 (1987)

[22] M. González-Melchor, F. Bresme and J. Alejandre, J. Chem. Phys. 122104710 (2005).

[23] E. Chacón and P. Tarazona, Phys. Rev. Lett. 91166103 (2003); P. Tarazona and E. Chacón, Phys. Rev. B. 70 235407 (2004).

[24] J. Chowdhary and B.M. Ladanyi, J. Phys. Chem. B 110 15442 (2006).

[25] E. Chacón, P. Tarazona and J. Alejandre, J. Chem. Phys. 125014709 (2006).

[26] See EPAPS Document No.[] for supplementary material.

[27] O. Esenturk and R.A. Walker, J. Chem. Phys. 125 174701 (2006). 